\section{Anti-IgE-Fertigspritze zur Asthmatherapie zugelassen}

Zur Behandlung von schwerem allergischem Asthma steht der lgE-Antikörper Omalizumab $\left(\right.$ Xolair $^{\odot}$ ) nicht mehr nur als lyophilisiertes Pulver, sondern auch als gebrauchsfertige Injektionslösung zur Verfügung. Die Vorteile erklärte Prof. Roland Buhl, Pneumologe, Mainz: „Bislang waren für die Behandlung mit Omalizumab 15- bis 20-minütige Aufbereitungszeiten nötig. Dank der Zulassung der gebrauchsfertigen Injektionslösung wird sowohl Patienten als auch uns Ärzten die Injektion deutlich vereinfacht." Studiendaten belegen, dass die Wirksamkeit der beiden Darreichungsformen vergleichbar und die pharmakologischen Parameter der gebrauchsfertigen Liquidformulierung der lyophilisierten Formulierung äquivalent sind. Die neue Fertigspritze ist in den Dosierungen 75 und 150 mg Omalizumab verfügbar. Dabei kann die invididuell benötigte Dosis durch Kombination der beiden Wirkstärken erzielt werden. Die neue Fertigspritze ermöglicht somit eine exaktere Dosierung.

Pressemeldung Novartis, 9.2.2011

\section{Alle Patientengruppen profitieren von Add-on mit Leukotrienantagonist}

In einer Subgruppenanlayse der zwölfmonatigen MONICA-Studie zum Langzeitnutzen der Zusatztherapie von Asthmapatienten mit Montelukast (Singulair ${ }^{\odot}$ ) konnte jetzt gezeigt werden, dass die Add-onTherapie mit dem Leukotrienrezeptorantagonisten die Asthmakontrolle und Lebensqualität in allen Patientengruppen verbessert (Virchow JC et al. Journal of Asthma 2010). Das Therapieergebnis, beurteilt mit dem fünf Fragen umfassenden ACT(asthma control test-)Score, verbessserte sich von 14,6 (Behandlungsbeginn) über 19,4 (nach 6 Monaten) auf 20,3 Punkte nach einem Jahr. Allerdings profitieren Asthmapatienten mit einer gleichzeitigen allergischen Rhinitis (AR) mehr von der Montelukast-therapie. Bei ihnen steig der ACT-Score, von 14,7 (Behandlungsbeginn) auf 21,0 Punkte nach 12 Monaten, bei Patienten ohne AR von 14,5 nur auf 19,3 Punkte. Dass unter 30-Jährige bessere Resultate der Asthmakontrolle zeigten als ältere Patienten, ist für Virchow und Kollegen ein möglicher Hinweis, dass Montelukast bereits im frühen Krankeitsstadium eingesetzt werden sollte.

\title{
PNEUMONEWS
}

\section{Herausgeber Klinik:}

Prof. Dr. A. Gillissen, Kassel

Prof. Dr. M. J. Kohlhäufl, Stuttgart-

Gerlingen

\section{Schriftleiter:}

Priv.-Doz. Dr. S. Budweiser, Rosenheim (Beatmungsmedizin)

Prof. Dr. G. Friedel, Stuttgart-Gerlingen (Thoraxchirurgie)

Prof. Dr. A. Ghofrani, Gießen, Dr. K. Gutjahr, Leipzig (Erkrankungen des Lungenkreislaufs)

Prof. Dr. A. Gillissen, Kassel (Asthma bronchiale)

Prof. Dr. M. Griese, München (Pädiatrische Pneumologie)

Prof. Dr. A. Günther, Prof. Dr. Ph. Markart, Gießen (Interstitielle Lungenerkrankungen)

Prof. Dr. M. J. Kohlhäufl, Stuttgart-Gerlingen (Tumoren)

Dr. S. R. Ott, Bern (Infektiologie)

Prof. Dr. K. Rasche, Wuppertal, Prof. Dr. K.-H. Rühle, Hagen, Prof. Dr. H. Schäfer,

Völklingen (Schlafmedizin)

Prof. Dr. G. Rohde, Maastricht/Niederlande (COPD, Emphysem)

Dr. K. Schultz, Bad Reichenhall (Rehabilitation)

Prof. Dr. M. Schwab, Prof. Dr. K. Mörike, Tübingen (Pharmakologische Therapie)

\section{Redaktion:}

Dr. Beate Schumacher (Chefredaktion, verantwortlich, Anschrift wie Verlag, beate.schumacher@springer.com) Ute Kempf (ute.kempf@springer.com), Dr. Judith Neumaier (judith.neumaier@t-online.de), Dr. Thomas Riedel, Angelika Holzgreve (Assistenz Chefredaktion, angelika.holzgreve@ springer.com)

\section{Verlag:}

Springer Medizin, ๑ Urban \& Vogel $\mathrm{GmbH}$, Aschauer Str. 30, D-81549 München, Tel.: 0 89/20 $3043-13$ 00, Fax: -13 99, http://www.springerfachmedien-medizin.de. Springer Medizin ist Teil der Fachverlagsgruppe Springer Science+Business Media.

\section{Geschäftsführer:}

Harm van Maanen, Ulrich Huber, Stephan Kröck, Dr. med. Esther Wieland, Matthias Wissel.

\section{Anzeigenverkauf:}

Ines Spankau (Anzeigenleitung, -1339, verantwortlich, Anschrift wie Verlag), Anzeigenpreisliste Nr. 4 vom 1. Oktober 2010.

Corporate Publishing:

Ulrike Hafner (06221/4878104).

\section{Herstellung:}

Christian Staral (Leitung); Schmidt Media Design, München (Layout);

Dieter L. Adam, München (Titelbild)

\section{Vertrieb/Marketing Services:}

Frank Niemann (Leitung, -14 11)

\section{Abonnement:}

Die Zeitschrift erscheint 6 x jährlich. Bezugspreise ab 2011: Einzelheft $12 €$ inkl. der deutschen MwSt. zuzügl. 2,50 € Versandkosten; Jahresabo $75 €$; Studenten/Ärzte in Aus- und Weiterbildung $60 €$, jeweils zuzügl. Versand (Inland $21 €$, Ausland $36 €)$.

Bestellungen nimmt der Verlag und jede Buchhandlung entgegen. Das Abonnement verlängert sich jeweils um ein Jahr, wenn es nicht zwei Monate vor Ende des Bezugszeitraums abbestellt wurde.

\section{Abonnentenservice:}

Tel.: 062 21/3 45-43 04; Fax: 062 21/3 45-42 29

Gesamtherstellung: Stürtz $\mathrm{GmbH}$, AlfredNobel-Str. 33, D-97080 Würzburg

Originalien: Zur Veröffentlichung kommen nur Arbeiten, die an anderer Stelle weder angeboten noch erschienen sind. Die Autoren sind verpflichtet zu prüfen, ob Urheberrechte Dritter berührt werden. Alle CME-Manuskripte werden von unabhängigen Experten begutachtet. Eine redaktionelle Bearbeitung bleibt vorbehalten. Autoren, die mit vollem Namen genannt sind, veröffentlichen ihre Beiträge in alleiniger Verantwortung.

Copyright: Der Verlag behält sich das ausschließliche Recht der Verbreitung, Übersetzung und jeglicher Wiedergabe auch von Teilen dieser Zeitschrift durch Nachdruck, Fotokopie, Mikrofilm, EDV-Verwertung, Funk- oder Fernsehaufzeichnung vor. Jede gewerblich hergestellte oder benutzte Fotokopie verpflichtet nach $\$ 54$ (2) UrHG zur Gebührenzahlung an die VG Wort, Abt. Wissenschaft, Goethestr. 49, D-80336 München, von der die Modalitäten zu erfragen sind.

Rechtseinräumung der Autoren: Mit der Einsendung eines Manuskripts zur Veröffentlichung überträgt der Verfasser dem Verlag für den Fall der Annahme das Recht, das Manuskript geändert oder unverändert, ganz oder teilweise in Pneumo-News und anderen Publikationen der Fachverlagsgruppe, in den zugehörigen Onlinediensten, in Onlinedatenbanken Dritter und, soweit vereinbart, gegen Nachhonorar in Sonderdrucken für Industriekunden zu nutzen.

Hinweis: Die in dieser Zeitschrift angegebe-nen Dosierungen - vor allem von Neuzulassungen sollten in jedem Fall mit dem Beipackzettel der verwendeten Medikamente verglichen werden. ISSN 1865-5467 


\section{Anti-IgE-Fertigspritze zur Asthmatherapie zugelassen}

Zur Behandlung von schwerem allergischem Asthma steht der lgE-Antikörper Omalizumab $\left(\right.$ Xolair $^{\odot}$ ) nicht mehr nur als lyophilisiertes Pulver, sondern auch als gebrauchsfertige Injektionslösung zur Verfügung. Die Vorteile erklärte Prof. Roland Buhl, Pneumologe, Mainz: „Bislang waren für die Behandlung mit Omalizumab 15- bis 20-minütige Aufbereitungszeiten nötig. Dank der Zulassung der gebrauchsfertigen Injektionslösung wird sowohl Patienten als auch uns Ärzten die Injektion deutlich vereinfacht." Studiendaten belegen, dass die Wirksamkeit der beiden Darreichungsformen vergleichbar und die pharmakologischen Parameter der gebrauchsfertigen Liquidformulierung der lyophilisierten Formulierung äquivalent sind. Die neue Fertigspritze ist in den Dosierungen 75 und 150 mg Omalizumab verfügbar. Dabei kann die invididuell benötigte Dosis durch Kombination der beiden Wirkstärken erzielt werden. Die neue Fertigspritze ermöglicht somit eine exaktere Dosierung.

Pressemeldung Novartis, 9.2.2011

\section{Alle Patientengruppen profitieren von Add-on mit Leukotrienantagonist}

In einer Subgruppenanlayse der zwölfmonatigen MONICA-Studie zum Langzeitnutzen der Zusatztherapie von Asthmapatienten mit Montelukast (Singulair ${ }^{\odot}$ ) konnte jetzt gezeigt werden, dass die Add-onTherapie mit dem Leukotrienrezeptorantagonisten die Asthmakontrolle und Lebensqualität in allen Patientengruppen verbessert (Virchow JC et al. Journal of Asthma 2010). Das Therapieergebnis, beurteilt mit dem fünf Fragen umfassenden ACT(asthma control test-)Score, verbessserte sich von 14,6 (Behandlungsbeginn) über 19,4 (nach 6 Monaten) auf 20,3 Punkte nach einem Jahr. Allerdings profitieren Asthmapatienten mit einer gleichzeitigen allergischen Rhinitis (AR) mehr von der Montelukast-therapie. Bei ihnen steig der ACT-Score, von 14,7 (Behandlungsbeginn) auf 21,0 Punkte nach 12 Monaten, bei Patienten ohne AR von 14,5 nur auf 19,3 Punkte. Dass unter 30-Jährige bessere Resultate der Asthmakontrolle zeigten als ältere Patienten, ist für Virchow und Kollegen ein möglicher Hinweis, dass Montelukast bereits im frühen Krankeitsstadium eingesetzt werden sollte.

\section{PNEUMONEWS}

\section{Herausgeber Klinik:}

Prof. Dr. A. Gillissen, Kassel

Prof. Dr. M. J. Kohlhäufl, Stuttgart-

Gerlingen

\section{Schriftleiter:}

Priv.-Doz. Dr. S. Budweiser, Rosenheim (Beatmungsmedizin)

Prof. Dr. G. Friedel, Stuttgart-Gerlingen (Thoraxchirurgie)

Prof. Dr. A. Ghofrani, Gießen, Dr. K. Gutjahr, Leipzig (Erkrankungen des Lungenkreislaufs)

Prof. Dr. A. Gillissen, Kassel (Asthma bronchiale)

Prof. Dr. M. Griese, München (Pädiatrische Pneumologie)

Prof. Dr. A. Günther, Prof. Dr. Ph. Markart, Gießen (Interstitielle Lungenerkrankungen)

Prof. Dr. M. J. Kohlhäufl, Stuttgart-Gerlingen (Tumoren)

Dr. S. R. Ott, Bern (Infektiologie)

Prof. Dr. K. Rasche, Wuppertal, Prof. Dr. K.-H. Rühle, Hagen, Prof. Dr. H. Schäfer,

Völklingen (Schlafmedizin)

Prof. Dr. G. Rohde, Maastricht/Niederlande (COPD, Emphysem)

Dr. K. Schultz, Bad Reichenhall (Rehabilitation)

Prof. Dr. M. Schwab, Prof. Dr. K. Mörike, Tübingen (Pharmakologische Therapie)

\section{Redaktion:}

Dr. Beate Schumacher (Chefredaktion, verantwortlich, Anschrift wie Verlag, beate.schumacher@springer.com) Ute Kempf (ute.kempf@springer.com), Dr. Judith Neumaier (judith.neumaier@t-online.de), Dr. Thomas Riedel, Angelika Holzgreve (Assistenz Chefredaktion, angelika.holzgreve@ springer.com)

\section{Verlag:}

Springer Medizin, ๑ Urban \& Vogel $\mathrm{GmbH}$, Aschauer Str. 30, D-81549 München, Tel.: 0 89/20 $3043-13$ 00, Fax: -13 99, http://www.springerfachmedien-medizin.de. Springer Medizin ist Teil der Fachverlagsgruppe Springer Science+Business Media.

\section{Geschäftsführer:}

Harm van Maanen, Ulrich Huber, Stephan Kröck, Dr. med. Esther Wieland, Matthias Wissel.

\section{Anzeigenverkauf:}

Ines Spankau (Anzeigenleitung, -1339, verantwortlich, Anschrift wie Verlag), Anzeigenpreisliste Nr. 4 vom 1. Oktober 2010.

Corporate Publishing:

Ulrike Hafner (06221/4878104).

\section{Herstellung:}

Christian Staral (Leitung); Schmidt Media Design, München (Layout);

Dieter L. Adam, München (Titelbild)

\section{Vertrieb/Marketing Services:}

Frank Niemann (Leitung, -14 11)

\section{Abonnement:}

Die Zeitschrift erscheint 6 x jährlich. Bezugspreise ab 2011: Einzelheft $12 €$ inkl. der deutschen MwSt. zuzügl. 2,50 € Versandkosten; Jahresabo $75 €$; Studenten/Ärzte in Aus- und Weiterbildung $60 €$, jeweils zuzügl. Versand (Inland $21 €$, Ausland $36 €)$.

Bestellungen nimmt der Verlag und jede Buchhandlung entgegen. Das Abonnement verlängert sich jeweils um ein Jahr, wenn es nicht zwei Monate vor Ende des Bezugszeitraums abbestellt wurde.

\section{Abonnentenservice:}

Tel.: 062 21/3 45-43 04; Fax: 062 21/3 45-42 29

Gesamtherstellung: Stürtz $\mathrm{GmbH}$, AlfredNobel-Str. 33, D-97080 Würzburg

Originalien: Zur Veröffentlichung kommen nur Arbeiten, die an anderer Stelle weder angeboten noch erschienen sind. Die Autoren sind verpflichtet zu prüfen, ob Urheberrechte Dritter berührt werden. Alle CME-Manuskripte werden von unabhängigen Experten begutachtet. Eine redaktionelle Bearbeitung bleibt vorbehalten. Autoren, die mit vollem Namen genannt sind, veröffentlichen ihre Beiträge in alleiniger Verantwortung.

Copyright: Der Verlag behält sich das ausschließliche Recht der Verbreitung, Übersetzung und jeglicher Wiedergabe auch von Teilen dieser Zeitschrift durch Nachdruck, Fotokopie, Mikrofilm, EDV-Verwertung, Funk- oder Fernsehaufzeichnung vor. Jede gewerblich hergestellte oder benutzte Fotokopie verpflichtet nach $\$ 54$ (2) UrHG zur Gebührenzahlung an die VG Wort, Abt. Wissenschaft, Goethestr. 49, D-80336 München, von der die Modalitäten zu erfragen sind.

Rechtseinräumung der Autoren: Mit der Einsendung eines Manuskripts zur Veröffentlichung überträgt der Verfasser dem Verlag für den Fall der Annahme das Recht, das Manuskript geändert oder unverändert, ganz oder teilweise in Pneumo-News und anderen Publikationen der Fachverlagsgruppe, in den zugehörigen Onlinediensten, in Onlinedatenbanken Dritter und, soweit vereinbart, gegen Nachhonorar in Sonderdrucken für Industriekunden zu nutzen.

Hinweis: Die in dieser Zeitschrift angegebe-nen Dosierungen - vor allem von Neuzulassungen sollten in jedem Fall mit dem Beipackzettel der verwendeten Medikamente verglichen werden. ISSN 1865-5467 